\title{
PROPERTIES OF TWO POINT BOUNDARY VALUE FUNCTIONS ${ }^{1}$
}

\author{
G. A. BOGAR ${ }^{2}$
}

1. Introduction. This paper is concerned with conditions under which certain functions arising from two point boundary value problems of quasi-differential equations are continuous and increasing.

We now define recursively an $n$th order linear differential operator by:

(1) $\mathfrak{H C}_{0}$ is the set of all real-valued continuous functions on $(-\infty, \infty)$ where $D_{0}(y)=y$ for $y$ an element of $\mathfrak{H}_{0}$, and

(2) for $1 \leqq k \leqq n$, let $\mathfrak{H}_{k}$ be the set of all $y$ in $\mathfrak{F C}_{k-1}$ which has a continuous derivative and $y$ an element of $\mathfrak{F}_{k}$

$$
D_{k}(y)=\frac{1}{f_{k, k+1}}\left[\left(D_{k-1}(y)\right)^{\prime}+\sum_{i=1}^{k-1} p_{k, i} D_{i-1}(y)\right]
$$

where $f_{k, k+1}$ is a continuous real-valued positive function on $(-\infty, \infty)$ and $p_{k, i}$ is a continuous function on $(-\infty, \infty)$.

The $n$th order quasi-differential equation to be studied is

$$
L_{n}[y]=\left(D_{n-1}(y)\right)^{\prime}+\sum_{i=1}^{n-1} p_{n, i} D_{i-1}(y)=0 .
$$

Quasi-differential equations similar to $\left(E_{n}\right)$ have also been studied by D. Hinton [1] and A. Zettl [6].

Before proceeding further we define the boundary value functions.

Definition 1.1. (a) For $t \in[a, \infty)$, and $i+j \geqq n$ define $z_{i j}(t)$ to be the greatest lower bound of the set of all $b>t$ such that $\left(E_{n}\right)$ has a nontrivial solution with a zero at $t$ of multiplicity at least $i$ and a zero at $b$ of multiplicity at least $j$.

(b) If $t \in[a, \infty)$ and $i+j \geqq n$ then $r_{i j}(t)$ is the greatest lower bound of the set of all $b>t$ such that there exists a nontrivial solution of $\left(E_{n}\right)$ which has a zero at some $t_{0} \geqq t$ of multiplicity at least $i$ and a zero at $b>t_{0}$ of multiplicity at least $j$.

Definition 1.2. For $t(-\infty, c]$ and $i+j \geqq n$, then $\bar{z}_{i j}(t)$ is the least upper bound of the set of all $b<t$ such that there exists a non-

Received by the editors November 29, 1968.

1 This paper is part of the author's dissertation. The work was supported, in part, by NASA Grant Number 43-001-029.

${ }^{2}$ The author wishes to thank Professor J. S. Bradley for his advice and assistance. The author is now a member of the staff at Montana State University. 
trivial solution $y$, which has a zero of multiplicity at least $i$ at $t$ and a zero at $b$ of multiplicity at least $j$.

Finally we define a class of operators which will be useful.

Definition 1.3. If $J$ is a subinterval of $(-\infty, \infty)$ denote by $T_{i j}[J]$ the class of operators $L_{n}$ for which $\left(E_{n}\right)$ has no nontrivial solution with at least $i$ zero at some $a \in J$ and at least $j$ zeros at some $b>a$, $b \in J,(i+j \geqq n)$. If $J$ is $(-\infty, \infty)$ we omit $J$.

2. Properties $z_{i j}(t)$ and $r_{i j}(t)$.

Definition 2.1. An extended real-valued function $\alpha$ is increasing if and only if, for any $s_{1}$ and $s_{2}$ such that $-\infty<s_{1}<s_{2}<\infty$, either $\alpha\left(s_{1}\right)<\alpha\left(s_{2}\right)$ or $\alpha\left(s_{2}\right)=\infty$.

T. Sherman [5] and D. Hinton [1] showed that $\eta_{1}(t)$, the first conjugate point of $t_{1}$ is an increasing function. J. Levin [2] stated without proof some conditions under which $r_{i j}(t)$ for the classical $n$th order equation is increasing. We now establish conditions under which $r_{i j}(t)$ and $z_{i j}(t)$ are increasing for $\left(E_{n}\right)$.

LeMma 2.1. If either $z_{i j}(t)$ or $r_{i j}(t)$ is an increasing function of $t$, then $z_{i j}(t)=r_{i j}(t)$, where $i+j=n$.

The proof of the lemma is a direct consequence of Definition 1.1 and the fact that the greatest lower bound can be replaced by the word minimum.

Definition 2.2. A fundamental set of solution $\left\{u_{\beta}(x, t)\right\}$ of $\left(E_{n}\right)$ is defined by $D_{\alpha}\left(u_{\beta}(t, t)\right)=\delta_{\alpha+1, \beta+1}(\alpha, \beta=0, \cdots, n-1)$. We also use the following notation:

$W\left(u_{i}(x, t), \cdots, u_{j}(x, t)\right)=\left|D_{\alpha}\left[u_{\beta}(x, t)\right]\right|, \quad i \leqq \beta \leqq j, 0 \leqq \alpha \leqq j-1$.

When $L_{2 n}$ is a selfadjoint operator, the next theorem shows that $z_{n n}(t)$ is an increasing function without placing further restrictions on $L_{2 n}$.

THEOREM 2.1. If $L_{2 n}[y]=0$ is a selfadjoint equation, then $z_{n n}(t)$ is increasing.

Proof. If $Z_{n n}(\alpha)<\infty$, then $z_{n n}(\beta) \leqq z_{n n}(\alpha)$ for $\beta<\alpha$ by Theorem 2.1 of W. T. Reid [4]. Assume now there exists $b>a$ such that $z_{n n}(a)$ $=z_{n n}(b)<\infty$. Then $z_{n n}(\alpha)=z_{n n}(a)$ for $\alpha \in[a, b]$ by the above and $W\left(u_{n}\left(x, z_{n n}(a)\right), \cdots, u_{2 n-1}\left(x, z_{n n}(a)\right)\right)=0$ for $x \in[a, b]$; but by Theorem 3.4 of D. Hinton [1] this cannot happen.

COROLlaRY 2.1. If $L_{2 n}[y]=0$ is a 2 nth order selfadjoint equation then $\bar{z}_{n n}(t)$ is increasing. 
Let $M_{+}\left(M_{-}\right)$be the set of all $t$ such that $z_{n n}(t)\left(\bar{z}_{n n}(t)\right)$ is finite.

CoROllaRY 2.2. For a selfadjoint 2 th order equation $\left(E_{n}\right), z_{n n}(t)$ restricted to $M_{+}$is continuous.

The proof follows exactly the same reasoning as in the proof of Corollary 3.5.2 of D. Hinton [1].

Now we consider conditions under which $r_{i j}(t)$ is increasing and continuous for the more general $n$th order operator $L_{n}$.

Lemma 2.2. If $L_{n} \in T_{k+1, n-k} \cap T_{k-1, n-k+1}$ for some $k \in\{1,2, \cdots, n-1\}$, $\tau<t$ and $W\left(u_{n-k}(\tau, t), \cdots, u_{n-1}(\tau, t)\right)=0$, then

$$
W^{\prime}\left(u_{n-k}(\tau, t), \cdots, u_{n-1}(\tau, t)\right) \neq 0 .
$$

Proof. Define constants $c_{n-k+i}$ by

$$
\begin{array}{r}
c_{n-k+i}=(-1)^{i} W\left(u_{n-k}(\tau, t), \cdots, u_{n-k+i-1}(\tau, t), u_{n-k+i+1}(\tau, t), \cdots, u_{n-1}(\tau, t)\right) \\
(i=1, \cdots, k-1) .
\end{array}
$$

Set

$$
y(x)=\sum_{i=n-k}^{n-1} c_{i} u_{i}(x, t) .
$$

Since $L_{n} \in T_{k-1, n-k+1}, c_{n-k}=W\left(u_{n-k+1}(\tau, t), \cdots, u_{n-1}(\tau, t)\right) \neq 0$ and the function $y$ given by $(2.1)$ is a nontrivial solution of $\left(E_{n}\right)$. We observe now that

$$
D_{l} y(\tau)=\sum_{i=n-k}^{n-1} c_{i} D_{l} u_{i}(\tau, t)=0, \quad 0 \leqq l \leqq k-2
$$

since $\sum_{i=n-k}^{n-1} c_{i} D_{l} u_{i}(\tau, t)$ is a determinant with two rows the same. Moreover $D_{k-1} y(\tau)=0$ since $D_{k-1} y(\tau)=W\left(u_{n-k}(\tau, t), \cdots, u_{n-1}(\tau, t)\right)$. For $1 \leqq k \leqq n-1$ we see that $W^{\prime}\left(u_{n-k}(\tau, t), \cdots, u_{n-k}(\tau, t)\right) \neq 0$, otherwise $D_{k}(y(\tau))=0$ which is a contradiction of the fact that $L \in T_{k+1, n-k}$.

CoRollary 2.3. If $L$ is selfadjoint $L \in T_{k-1, n-k} \cap T_{k-1, n-k+1}$ for some $k, \tau>t$ and $W\left(u_{n-k}(\tau, t), \cdots, u_{n-1}(\tau, t)\right)=0$ then $W^{\prime}\left(u_{n-k}(\tau, t), \cdots, u_{n-1}(\tau, t)\right) \neq 0$ for $\tau>t$.

The proof follows easily from Lemma 2.2 and Theorem 4.2 of D. B. Hinton [1].

Definition 2.3. Denote by $N_{k, n-k}$ the class of operators for which $r_{k, n-k}(t)<\min \left(r_{k+1, n-k}(t), r_{k-1, n-k+1}(t)\right)$ if $r_{k, n-k}(t)$ is finite, $-\infty<t<\infty$.

Theorem 2.2. If $L_{n}$ is an element of $N_{k, n-k}$ for some $k \in\{1,2, \cdots, n-1\}$ then $r_{k, n-k}(t)$ is an increasing function of $t$. 
ProOF. First we observe that $r_{k, n-k}(t)$ is nondecreasing by definition. If there exists $\gamma$ in $(-\infty, \infty)$ such that $r_{k, n-k}(\gamma)=\infty$ then $r_{k, n-k}(t)$ is increasing on $[\gamma, \infty]$. Also if $c>d$ where $r_{k, n-k}(d)<\infty$ and $r_{k, n-k}(c)=\infty$, then $r_{k, n-k}(d)<r_{k, n-k}(c)$.

In order to complete the proof we need only show $z_{k, n-k}(t)$ $=r_{k, n-k}(t)$, when $r_{k, n-k}(t)<\infty$. From the definition and the fact that greatest lower bound can be replaced by minimum we know there exists a nontrivial solution $y$ of $L$ which has a zero of multiplicity $k$ at $\tau \in\left[t, r_{k, n-k}(t)\right)$ and a zero of multiplicity $n-k$ at $r_{k, n-k}(t)$. In order to simplify notation we will use $\beta$ for $r_{k, n-k}(t)$ in the remainder of the proof. This solution $y$ can be written as

$$
y(x)=c_{n-k} u_{n-k}(x, \beta)+\cdots+c_{n-1} u_{n-1}(x, \beta),
$$

and since $y$ has a zero of multiplicity $k$ at $\tau$,

$$
W\left(u_{n-k}(\tau, \beta), \cdots, u_{n-1}(\tau, \beta)\right)=0 .
$$

It follows from the continuous dependence of solutions upon initial values and the fact that a determinate is a continuous function of its elements that given $\epsilon>0$ and $i \in\{0,1, \cdots, n-1\}$, then there exists $\delta_{\epsilon}>0$ such that $0<\beta-\beta_{\epsilon}<\delta_{\epsilon}$ implies that

$$
\left|W\left(u_{i}(x, \beta), \cdots, u_{n-1}(x, \beta)\right)-W\left(u_{i}\left(x, \beta_{\epsilon}\right), \cdots, u_{n-1}\left(x, \beta_{\epsilon}\right)\right)\right|<\epsilon
$$

for all $x \in[t, \beta]$.

Now for $\tau \neq t$, choose $\epsilon<(t-\tau) / 2, \delta=\min \left\{\delta_{\epsilon},(\tau-t) / 4,(\beta-t) / 4, \epsilon\right\}$ and $\beta_{\epsilon} \in(\beta-\delta, \delta)$. Since $r_{k, n-k}(t)<\min \left(r_{k+1, n-k}(t), r_{k-1, n-k+1}(t)\right)$ then $L$ is an element of $T_{k+1, n-k} \cap T_{k-1, n-k+1}$ on $[t, \beta]$. By Lemma 2.2, we know that $W^{\prime}\left(u_{n-k}(\tau, \beta), \cdots, u_{n-1}(\tau, \beta)\right) \neq 0$. But

$$
W\left(u_{n-k}(\tau, \beta), \cdots, u_{n-1}(\tau, \beta)\right)=0
$$

and thus it follows that for some $x \in(\tau-\epsilon, \tau+\epsilon)$

$$
W\left(u_{n-k}\left(x, \beta_{\epsilon}\right), \cdots, u_{n-1}\left(x, \beta_{\epsilon}\right)\right)=0 .
$$

Hence there exists a nontrivial solution $y$, which has a zero of order $n-k$ at $\beta_{\epsilon}<\beta$ and a zero of order $k$ at a point $\tau>t$, which is a contradiction. Hence $t=\tau$ and the proof is complete.

Corollary 2.4. Let $L[y]=L_{0}[y]+p y$ where $L_{0} \in T_{0}$ and $p(t)$ $>0(<0)$. Then $r_{k, n-k}(t)$ is increasing.

The proof follows from Theorem 2.3 and Theorem 3.2 of $Z$. Nehari [3].

By strengthening the hypothesis of Theorem 2.3 we now give a condition under which $r_{k, n-k}(t)$ is both increasing and continuous. 
First let $S_{k}=\left\{t: r_{k, n-k}(t)\right.$ is finite $\}$.

THEOREM 2.3. If $r_{k, n-k}(t)<\min \left\{r_{k+1, n-k}(t), r_{k-1, n-k+1}(t)\right\}$ for some $k \in\{1,2, \cdots, n-1\}$, then $S_{k}$ is an interval and $r_{k, n-k}(t)$ is continuous on $S_{k}$.

\section{REFERENCES}

1. D. B. Hinton, Disconjugate properties of a system of differential equations, J. Differential Equations 2 (1966), 420-437.

2. A. Ju Levin, On the distributions of the zeros of solutions of a linear differential equation, Dokl. Akad. Nauk SSSR 156 (1964), 1281-1284=Soviet Math. Dokl. 5 (1964), 818-821 (Russian).

3. Z. Nehari, Disconjugate linear operators, Trans. Amer. Math. Soc. 129 (1967), 500-516.

4. W. T. Reid, Oscillation criteria for linear differential systems with complex coefficients, Pacific J. Math. 6 (1956), 733-751.

5. T. L. Sherman, Properties of $n$th order linear differential equations, Pacific J. Math. 15 (1965), 1045-1060.

6. A. J. Zettl, Adjoint linear differential operators, Proc. Amer. Math. Soc. 16 (1965), 1239-1241.

Montana State University 